\title{
An experimental study to evaluate the shade change of reactive dyed woven fabric after application of water repellent finish and cross linker
}

\begin{abstract}
Finishing is the last stage of textile chemical wet processing to impart aesthetics and functional characteristics in woven and knitted fabrics. Water repellent finishes form a thin hydrophobic layer on the surface of the cotton fabric, which changes its hydrophilic nature to hydrophobic. Water repellent finishes influences on the color shades and other properties of dyed finished fabric. However, despite its significance for the textile processing industry, the amount of color change is yet to be determined quantitatively. In this backdrop, the present research work has been carried out to predict the amount of color change as affected by different concentrations of water repellent finishes and cross linkers. The results show that oil and water repellent finishes have a significant impact on shade depth of reactive dyed fabric. These finishes increase the $\mathrm{K} / \mathrm{S}$ value in dark shades and decreases in light and medium shades. The results show that the significant color change occur after application of finish.
\end{abstract}

Keywords: water repellent finish, cross linker, softener, shade change, contact angle
Volume 3 Issue 2 - 2017

\author{
Assad Farooq, Kanwal Imtiaz, Farida Irshad, \\ Muhammad Azeem Ashraf \\ Department of Fibre and Textile Technology, University of \\ Agriculture, Pakistan
}

Correspondence: Dr.Assad Farooq, Department of Fibre and Textile Technology, University of Agriculture, Faisalabad, Pakistan, Tel 924 1920 1267, Email assadfarooq@googlemail.com

Received: July 19, 2017 | Published: November 06, 2017

\section{Introduction}

Cotton is a natural fiber having water loving nature due to presence of three -OH groups in its chemical structure. These hydroxyl groups show great affinity for water and other chemicals which has made the processing of cotton easier. Higher moisture regain of cotton provides the comfort to wearer. ${ }^{1}$ Finishes which provide the quality of oil-water repellency are very significant in all parts of the home and technical textile's market. It's so easy to get water repellency by applying various products like silicones, higher fatty acid groups whether the oil repellency can only be taken with fluorocarbon polymers. This is very interesting new development of chemical finishing. Repellent finishes can also provide good durable press properties, easier ironing and drying, increased resistance to all kinds of chemicals like acids \&alkali's. ${ }^{2}$

By decreasing the surface energy of fibers, repellent properties are attained. The purpose of the water repellent finish is to make a fine water hating layer on the surface of fabric. This procedure works on the principle of adhesive interaction of fiber \& liquid and inner cohesive interactions with-in the liquid. ${ }^{3}$ Now, if the adhesive attractions between the liquid and the fiber is lower than the cohesive interactions within the liquid, the water drop will not spread over the fabric. Fluorocarbons are the mostly used oleo-phobic and hydrophobic finishes having the property of very low interfacial tension towards all the chemicals. ${ }^{4}$

To get the best durability of the oil and water repellent finishes, good fabric preparation is essential. Textile auxiliary residues let's say surfactants, pretreatment agents, dyeing auxiliaries and sizes on the fabric can affect the performance of oil \& water repellent finishes, as well as the wash fastness properties. ${ }^{5}$ It is common fact that the water repellency of fluorocarbon based finishes lowers with continuous washing and recovers with subsequent heat treatments. However, the reason for this lowered water repellency is not so clear, it has been said that that flouro-alkyl groups rotate in the polymer substrate to restrain the water loving condition during washing. Now, the concept of easy care has been extended to include the properties of oil and water repellency in clothing and upholstery applications. The approach of easy care covers both the easy to clean fabrics and no necessity to the iron clothes on everyday basis. ${ }^{6}$ On account of easy care finishes, cross linkers play a pivoted role to increase the washing durability of repellent finishes but it can harden the feel of fabric to compensate which softener is added in recipe ${ }^{7}$.

Change in mechanical properties and color variations take place when finishes are applied on the surface of dyed textile fabric. Color change due to various finishes has remained the biggest issue for decades. So, there is a dire need of experimentation to evaluate the shade change after application of finish. Current research work has been carried out to predict the shade change after application of water replant finishes on reactive dyed woven fabric.

\section{Materials and methods}

Scoured and bleached $100 \%$ cotton plain woven fabric was used for the experimental work as shown in Table 1. Fabric samples were dyed by following Pad-Dry-Cure method and by using the chemicals as given in Table 2. For the fixation of reactive dye molecules on the surface of fabric drying was done at $1200^{\circ} \mathrm{C}$ for 3 minutes and curing was performed at $1600^{\circ} \mathrm{C}$ for 2 minutes. The samples were dyed in three different colors, i.e., red, blue and yellow. By varying the dye concentrations dark, medium and light shades were achieved in all three colors.

After dyeing, finishing solutions were prepared by using different concentrations of Oil-water repellent finishes and cross linkers as shown in Table 3. Impregnation of finish was done by setting the rollers of padder at $75 \%$ pick up. The impregnated samples were dried at $1000^{\circ} \mathrm{C}$ for 3 minutes and cured at $1500^{\circ} \mathrm{C}$ for 5 minutes that 
allowed the fixation of finish. In current research color was assessed by through spectrophotometer (Data colour SF-600) for Shade depth $(\mathrm{K} / \mathrm{S}$ value) and the contact angle between the water and fabric surface was measured by using Drop Shape Analysis System DSA 30.

Table I Fabric Construction

\begin{tabular}{lllll}
\hline $\begin{array}{l}\text { Fabric } \\
\text { type }\end{array}$ & Thickness $(\mathbf{m m})$ & $\begin{array}{l}\text { Picks/ } \\
\text { Inch }\end{array}$ & $\begin{array}{l}\text { Ends/ } \\
\text { Inch }\end{array}$ & $\begin{array}{l}\text { Fabric } \\
\text { Weight }\left(\mathbf{g} / \mathbf{m}^{2}\right)\end{array}$ \\
\hline $\begin{array}{l}\text { Plain } \\
\text { Fabric }\end{array}$ & 5.4 & 68 & 136 & 150 \\
\hline
\end{tabular}

Table 2 Recipe for dyeing

\begin{tabular}{ll}
\hline Chemicals & Concentration \\
\hline Shade depths & $1.5 \%, 1 \%, 0.5 \%$ \\
Water & $91 \%$ \\
Sodium Sulphate & $6 \mathrm{~g}$ \\
Sodium Carbonate & $2 \mathrm{~g}$ \\
Wetting agent & $0.12 \mathrm{~g}$ \\
\hline
\end{tabular}

Table 3 Different concentrations of finish and cross linker

\begin{tabular}{llll}
\hline Colors & Shades & $\begin{array}{l}\text { Water repellent } \\
\text { finish conc. }\end{array}$ & Cross linker conc. \\
\hline Red & Light & $2 \%$ & $5 \%$ \\
Blue & Medium & $4 \%$ & $10 \%$ \\
Yellow & Dark & &
\end{tabular}

\section{Contact angle measurement}

The shape of drop that it assumes on the fabric surface is called its wet ability. Treated fabric surface is evaluated by measuring its contact angle towards water. Specimen is fitted on the stage to measure the contact angle and then water drop is placed on the fabric surface. Drop shape analysis system has optics with manual or software-controlled zoom and standard or high speed camera, which help to capture the picture and computer interface, give assessment of contact angle.

\section{Shade depth measurement (Data color SF-600)}

Spectrophotometer works on three basic standards a light source, a source to direct the light on the sample and to catch the reflected light and a spectrometer that regulates the intensity of received light as a function of wavelength. It measures the extent of light, a sample (liquid or solid) absorbs. Light is passed through the sample and a detector measures the intensity of that light that passes through the sample and smashes that detector. The ray of light consists of photons (energy packets), and when these packets encounter an analyte molecule (an analyte being the molecule that is under study), there's a chance that this analyte absorbs the energy present in photon. This absorption consequently reduces the number of photons in the light beam and thus reduces the intensity of that light beam.

\section{Result and discussions}

\section{Contact angle measurements}

The results of the contact angle of the samples treated with Oil water repellent finishes and cross linkers on various shades of red, yellow and blue reactive dyes are depicted in Figures 1-3 respectively. It can be seen that different concentrations of oil and water repellent finish and cross linking agent produce different contact angle values on all different shades of reactive dyed fabric. An increase in oil water repellent finishes and cross linker concentrations provide the better repellency on account of higher value of contact angle. Contact angle value is specifically attributed to Oil Water repellent finish concentration higher the concentration, higher will be the water repellency. As with 2\% finish and 5\% cross linker the contact between water drop and fabric surface was lesser as compared to the fabric treated with higher concentrations $4 \%$ finish and 5\% cross linker for all reactive dyed colors and shades. The same trend was observed with varying concentrations of oil water repellent finish $2 \%, 4 \%$ and $10 \%$ cross linker. It can be observed that cross linker concentration does not affect the contact angle measurements significantly as it only plays its role to enhance the durability and performance of oil-water repellent finish Moreover, different dyes concentrations also ineffectual on repellency of fabric. So, it is only affected by the concentration of repellent finish.

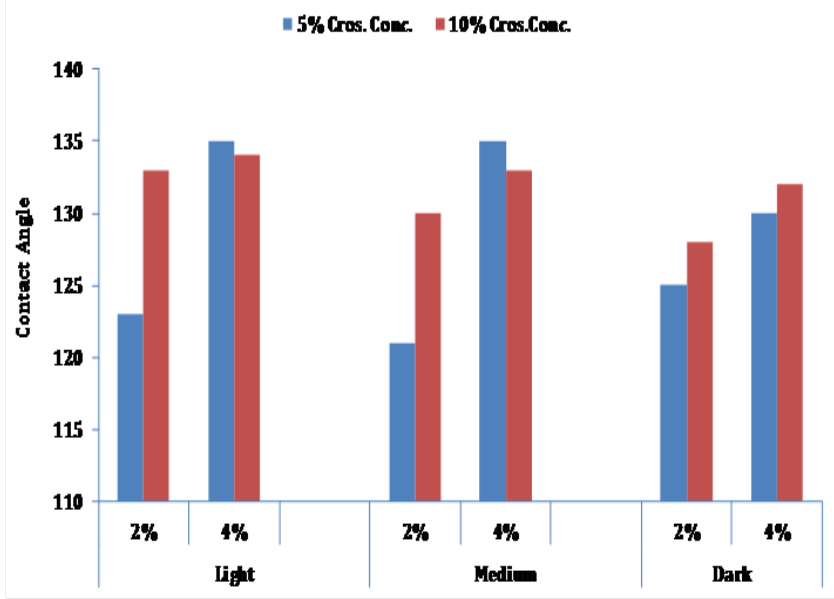

Figure I Contact Angle for Reactive Red.

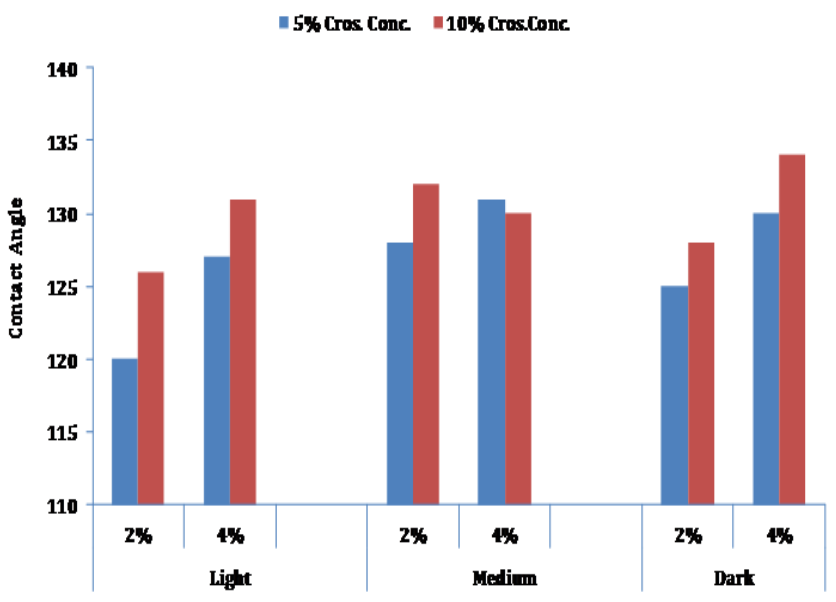

Figure 2 Contact Angle for Reactive Yellow.

\section{Shade depth}

The graphical representation of shade depth at different concentrations of reactive dye and finishes is given in Figures 4-6. Comparison of shade depth values before and after application of Oil 
water repellent finish and cross linker indicates that shade depth value increases in dark shades while decreases in light and medium shades after application of finish. It is due to high curing temperature and reaction between the repellent finish chemicals and dyes molecules.

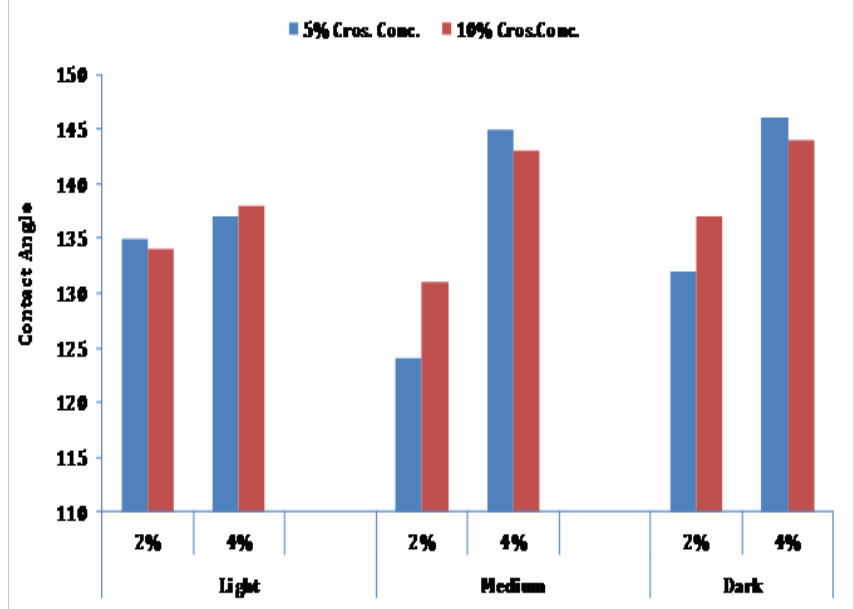

Figure 3 Contact Angle for Reactive Blue.

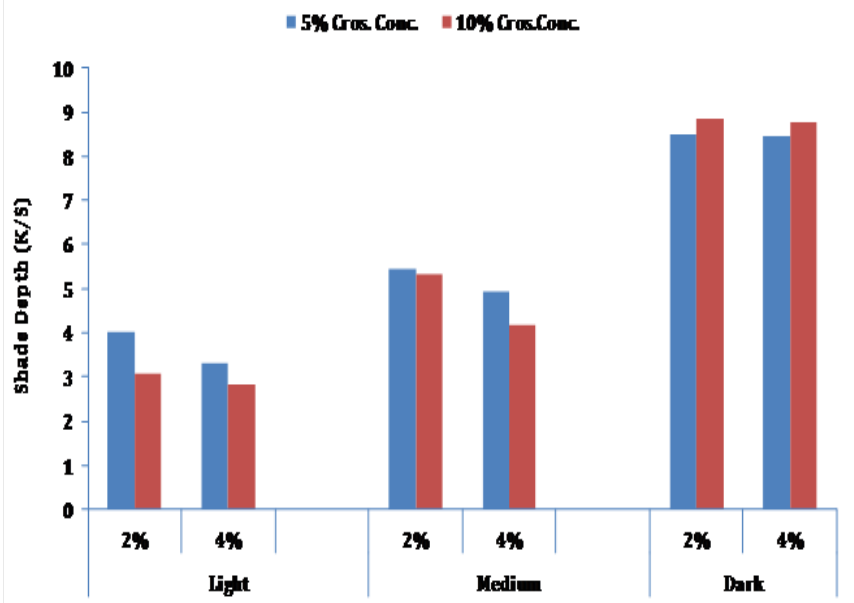

Figure 4 Shade Depth for Reactive Red.

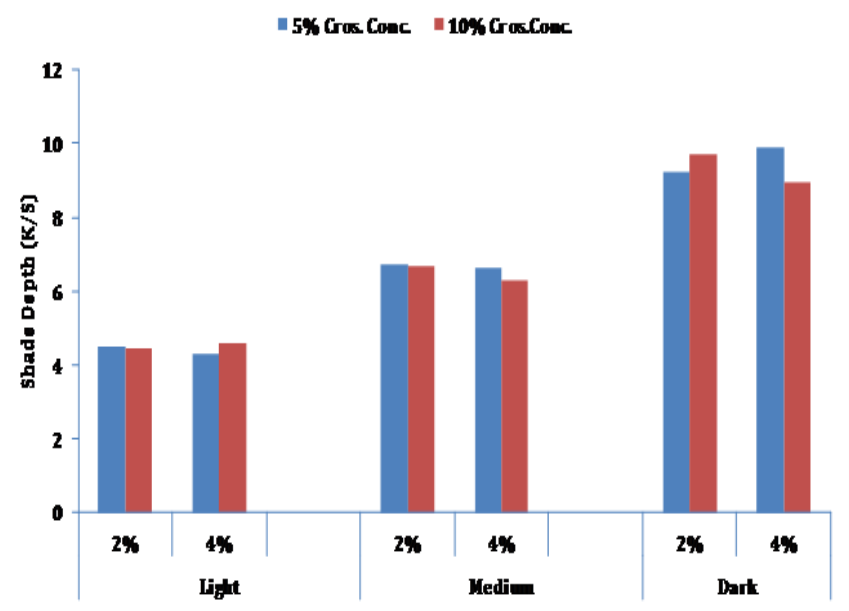

Figure 5 Shade Depth for Reactive Yellow.

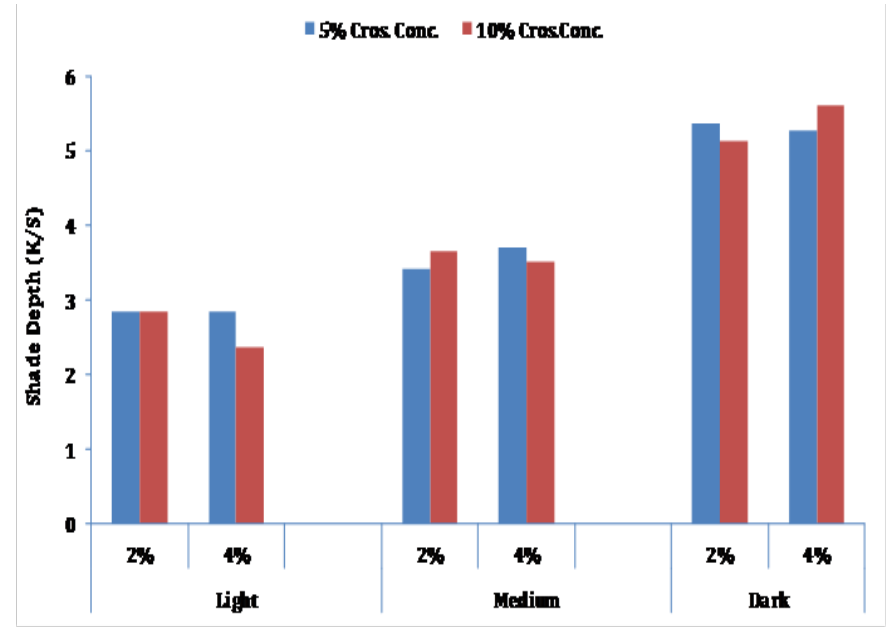

Figure 6 Shade Depth for Reactive Blue.

\section{Conclusion}

Knowledge of the relationship between chemicals and shade change of fabric assists the producers to arrange the chemical formulations. This will allow a producer to make an informed decision that will account for the required shade of the fabric at the best finish usage. In this study, shade change of reactive dyed fabric after application of oil water repellent finish was evaluated. Results proved that by application of finish oil and water repellency can be achieved successfully. However, finishes cause the change in shade depth of different primary colors. It increases the $\mathrm{K} / \mathrm{S}$ value in dark shades and reduces in light and medium shades.

\section{Acknowledgments}

Authors express their deep sense of gratitude to Higher Education Commission of Pakistan for financially supporting this project work under Technology Development Fund.

\section{Conflict of interest}

a. The manuscript is based on facts and has not been published before.

b. All financial support has been listed in the paper. No other funding has been provided for this manuscript.

\section{References}

1. Kang IS, Yang CQ, Wei W, et al. Mechanical strength of durable press finished cotton fabrics: Part I: Effects of acid degradation and crosslinking of cellulose by polycarboxylic acids. Textile Research $J$. 1998;68(11):86-870

2. Schindler WD, Hauser PJ. Chemical finishing of textiles. 2004.

3. Namligoz ES, Bahtiyari MI, Hosaf E, et al. Performance comparison of new (dendrimer, nanoproduct) and conventional water, oil and stain repellents. Fibers \& Textiles in Eastern Europe. 2009;17(5):76-81.

4. Elgarhy Y. Enhancement of durable soil release and soil resist, stain resist water and oil repellency and the softness of fibrous substrates, the substrates so treated and the treating composition. US Patent 7329367 , Trichromatic Carpet Inc, Canada; 2008. 
5. Brewer SA, Willis CR. Structure and oil repellency: textiles with liquid repellency to hexane. Applied Surface Science. 2008;254(20):6450-6454

6. Brodmann GL. Performance of nonformaldehyde cellulose reactants. AATCC Review. 1990;22(11):13-16.
7. Chen CC. Crosslinking of Cotton Cellulose in the Presence of Mixed Catalysts. Textile Research J. 1989;59(8):464-469. 